MPI-Ph/92-38

LPTB 92-6

\title{
Relating Weyl and diffeomorphism anomalies on super Riemann surfaces
}

\author{
Jean-Pierre Ader ${ }^{a \S}, \quad$ François Gieres ${ }^{b \dagger}$, Yves Noirot $^{a}$ \\ ${ }^{a}$ Laboratoire de Physique Théorique \\ Université de Bordeaux I \\ Rue du Solarium \\ F - 33170 - Gradignan \\ ${ }^{b}$ Max-Planck-Institut für Physik \\ Werner-Heisenberg-Institut \\ Föhringer Ring 6 \\ D - 8000 - München 40
}

\begin{abstract}
Starting from the Wess-Zumino action associated to the super Weyl anomaly, we determine the local counterterm which allows to pass from this anomaly to the chirally split superdiffeomorphism anomaly (as defined on a compact super Riemann surface without boundary). The counterterm involves the graded extension of the Verlinde functional and the results can be applied to the study of holomorphic factorization of partition functions in superconformal field theory.
\end{abstract}

$\S$ E-mail address: ADER@FRCPN11.

$\dagger$ Alexander von Humboldt Fellow. E-mail address: FRG@DM0MPI11.

$\ddagger$ Unité Associée au CNRS, U.A. 764.

MPI-Ph/92-38

LPTB 92-6

April 1992 


\section{Introduction}

Quite recently, the authors of reference [1] have determined the local counterterm to the effective action which allows one to shift the Weyl anomaly to the factorized (i.e. chirally split) diffeomorphism anomaly (as defined on an arbitrary compact Riemann surface without boundary). This result was then explored [2] to prove the existence and holomorphic factorization of partition functions viewed as functionals of Beltrami coefficients (the latter parametrizing the space of conformal structures). A natural question to ask is whether these results also hold in superconformal field theory and in the present paper, we address these issues and extend the analysis [1] in a supersymmetric way.

Let us be more concrete and present an outline of the paper. The arena we work on is a compact super Riemann surface (SRS) $\mathbf{S} \boldsymbol{\Sigma}$ without boundary [4]. We start from the super Weyl anomaly, $\mathcal{A}_{W}[\Omega]$, which depends on the supervielbein fields and on the Weyl ghost $\Omega$ [5] [6]. Furthermore, we consider the chirally split form of the superdiffeomorphism anomaly, $\mathcal{A}_{D}\left[C^{z}\right]+$ c.c., which depends on the super Beltrami coefficients and on the ghosts $C^{z}, C^{\bar{z}}$ parametrizing supercoordinate transformations [3] [7]. The main goal is to determine the local functional $\Gamma_{l o c}$ whose BRS variation $s \Gamma_{l o c}$ relates these anomalies (thus proving their equivalence):

$$
\mathcal{A}_{W}[\Omega]+s \Gamma_{\text {loc }}=\mathcal{A}_{D}\left[C^{z}\right]+\text { c.c. } .
$$

We start from the Wess-Zumino (WZ) action for the super Weyl anomaly [6] and successively construct the three local counterterms contributing to $\Gamma_{l o c}$. The first and third of these terms represent the supersymmetric generalization of the Liouville counterterm and of the Verlinde action [8] [1], respectively. The latter functional admits various applications in conformal field theory [8] [1] [2] and plays a rôle for the field-theoretical realization of $W$-algebras [9].

While the construction of the super Liouville counterterm does not pose any major problems, the remainder of the calculations is technically very complicated. For this reason, the corresponding details have been deferred to appendix C. Remarkably enough, the results take a compact and transparent form after their projection to component fields in the WZ-gauge (section 9). We conclude by commenting on the applications of the results to superconformal field theory.

In appendices A and B, we elaborate on the approach [10 to SRS's and superconformal models; the corresponding results are applied in the main body of the text and are of independent interest as a complement to the study [10]. Although we rely on the latter work for the parametrization of superconformal structures, we would like to mention that other approaches have previously been considered by various groups [11- 13].

\footnotetext{
${ }^{1}$ Actually, we will only consider supercomplex structures satisfying $H_{\theta}{ }^{z}=0$ - see section 3 - because the chirally split superdiffeomorphism anomaly is only known in this case for higher genus surfaces [3].
} 
As to our notation, we label the local coordinates on $\mathbf{S} \boldsymbol{\Sigma}$ by $(z, \bar{z}, \theta, \bar{\theta})$. The canonical basis of the tangent and cotangent spaces are, respectively, denoted by

$$
D \equiv D_{\theta} \equiv \partial_{\theta}+\theta \partial_{z} \quad, \quad D^{2}=\partial \equiv \partial_{z} \quad \text { and c.c. }
$$

and by

$$
e^{z} \equiv d z+\theta d \theta \quad, \quad e^{\theta} \equiv d \theta \quad \text { and c.c. . }
$$

In conclusion, we recall that the canonical 1-forms $e^{z}$ and $e^{\theta}$ satisfy the structure equations

$$
d e^{z}=-e^{\theta} e^{\theta} \quad, \quad d e^{\theta}=0
$$

\section{Vielbein fields and super Weyl anomaly}

The basic variables of supergravity are the supervielbein 1-forms $E^{A}$ [14]. In a light-cone type basis in two dimensions, we have $\left(E^{A}\right)=\left(E^{++}, E^{--}, E^{+}, E^{-}\right)$ which quantities can be expressed in terms of super isothermal coordinates $(Z, \bar{Z}, \Theta, \bar{\Theta})$, e.g. for the spatial components

$$
\begin{aligned}
& E^{++}=e^{Z}\left[\delta_{\bar{Z}}^{++} \exp (\sigma)\right] \equiv e^{Z} \rho_{Z} \\
& E^{--}=e^{\bar{Z}}\left[\delta_{\bar{Z}}^{--} \exp (\sigma)\right] \equiv e^{\bar{Z}} \rho_{\bar{Z}} .
\end{aligned}
$$

Here, $e^{Z}$ and $e^{\bar{Z}}$ are the canonical 1-forms and $\sigma$ is a scalar superfield 15. Thus, the "supermetric" 2 is given by

$$
d s^{2}=E^{++} E^{--}=e^{Z} e^{\bar{Z}}\left(\rho_{Z} \rho_{\bar{Z}}\right) \equiv e^{Z} e^{\bar{Z}} \rho_{Z \bar{Z}}
$$

Under an infinitesimal super Weyl rescaling with parameter $\Omega$, the conformal factors $\rho_{Z}$ and $\rho_{\bar{Z}}$ transform according to $\delta_{\Omega} \rho_{Z}=\Omega \rho_{Z}, \delta_{\Omega} \rho_{\bar{Z}}=\Omega \rho_{\bar{Z}}$.

The only independent field strength of the theory is a scalar superfield $S$ containing the scalar curvature of space-time among its components. The field $S$ transforms as [15]

$$
\delta_{\Omega} S=-\Omega S-2 \nabla_{+} \nabla_{-} \Omega,
$$

where $\nabla_{+}$and $\nabla_{-}$are the spinorial components of the Lorentz-covariant derivative in superspace.

The super Weyl anomaly $\mathcal{A}_{W}$ and the associated $W Z$ action $\Gamma_{W}$ have the form [6]

$$
\begin{aligned}
\mathcal{A}_{W}[\Omega, E] & \equiv K \int_{\mathbf{S} \boldsymbol{\Sigma}} d^{4} z E \Omega S \\
\Gamma_{W}[\phi, E] & =K \int_{\mathbf{S} \mathbf{\Sigma}} d^{4} z E\left\{\left(\nabla_{+} \phi\right)\left(\nabla_{-} \phi\right)+S \phi\right\} \\
\delta_{\Omega} \Gamma_{W}[\phi, E] & =-\mathcal{A}_{W}[\Omega, E] .
\end{aligned}
$$

\footnotetext{
${ }^{2}$ For the definition and interpretation of a supermetric, see the remarks in references [16].
} 
Here, $K$ is a constant, $E \equiv \operatorname{sdet}\left(E_{M}^{A}\right)$ and $\phi$ is the Goldstone superfield transforming as $\delta_{\Omega} \phi=-\Omega$.

If we express the functionals (6) in terms of isothermal coordinates, we obtain

$$
\begin{aligned}
\mathcal{A}_{W}[\Omega, E] & =-K \int_{\mathbf{S} \boldsymbol{\Sigma}} d^{4} Z \Omega D_{\Theta} D_{\bar{\Theta}} \ln \rho_{Z \bar{Z}} \\
\Gamma_{W}[\phi, E] & =-K \int_{\mathbf{S} \boldsymbol{\Sigma}} d^{4} Z\left\{\phi\left(D_{\Theta} D_{\bar{\Theta}} \phi\right)+\phi\left(D_{\Theta} D_{\bar{\Theta}} \ln \rho_{Z \bar{Z}}\right)\right\}
\end{aligned}
$$

where $D_{\Theta} \equiv \partial_{\Theta}+\Theta \partial_{Z}$ and $D_{\bar{\Theta}} \equiv \overline{D_{\Theta}}$ belong to the canonical tangent space basis.

\section{Beltrami coefficients and superdiffeomor- phism anomaly}

An atlas of superconformal coordinates $(Z, \bar{Z}, \Theta, \bar{\Theta})$ on $\mathbf{S} \boldsymbol{\Sigma}$ defines a supercomplex structure or, equivalently, a superconformal class of vielbein fields. These structures are parametrized by Beltrami coefficients $H_{\bar{\theta}}{ }^{z}, H_{\theta}{ }^{z}$ (and c.c.), see [10] and references therein. The parametrization is described by choosing a reference coordinate system, denoted by small coordinates $(z, \bar{z}, \theta, \bar{\theta})$, and expressing the canonical 1-forms of the coordinate system $(Z, \bar{Z}, \Theta, \bar{\Theta})$ with respect to the corresponding 1 -forms of the reference coordinate system [10]:

$$
\begin{aligned}
e^{Z} & =\left[e^{z}+e^{\bar{z}} H_{\bar{z}}{ }^{z}+e^{\theta} H_{\theta}{ }^{z}+e^{\bar{\theta}} H_{\bar{\theta}}{ }^{z}\right] \Lambda_{z}{ }^{Z} \\
e^{\Theta} & =\left[e^{z}+e^{\bar{z}} H_{\bar{z}}{ }^{z}+e^{\theta} H_{\theta}{ }^{z}+e^{\bar{\theta}} H_{\bar{\theta}}{ }^{z}\right] \tau_{z}{ }^{\Theta}+\left[e^{\theta} H_{\theta}{ }^{\theta}+e^{\bar{z}} H_{\bar{z}}{ }^{\theta}+e^{\bar{\theta}} H_{\bar{\theta}}{ }^{\theta}\right] \sqrt{\Lambda_{z}{ }^{Z}}
\end{aligned}
$$

(and c.c.). By fiat, the ' $H$ ' are inert under super Weyl transformations. The structure relations for $e^{Z}, e^{\bar{Z}}, e^{\Theta}, e^{\bar{\Theta}}$ ( i.e. eqs.(四) as written in terms of capital coordinates) imply that all the ' $H$ ' depend only on two independent ones, namely $H_{\bar{\theta}}{ }^{z}, H_{\theta}{ }^{z}$ (and c.c.). Moreover, these relations imply that $\Lambda$ is an independent factor (satisfying a linear differential equation given below) and that $\tau$ depends on it and on the ' $H$ '.

As discussed in reference [10], the restriction of the geometry where $H_{\theta}{ }^{z}=$ 0 (and c.c.) is compatible with superconformal changes of coordinates, but it implies that the superdiffeomorphism group has to be restricted to a subgroup leaving this equation invariant. At the infinitesimal level, this restriction is given by the relation

$$
C^{\theta}=\frac{1}{2} D C^{z} \quad \text { and c.c. } \quad
$$

where

$$
\begin{aligned}
C^{z} & \equiv \Xi^{z}+\Xi^{\bar{z}} H_{\bar{z}}^{z}+\Xi^{\theta} H_{\theta}{ }^{z}+\Xi^{\bar{\theta}} H_{\bar{\theta}}^{z} \\
C^{\theta} & \equiv \Xi^{\theta} H_{\theta}{ }^{\theta}+\Xi^{\bar{z}} H_{\bar{z}}{ }^{\theta}+\Xi^{\bar{\theta}} H_{\bar{\theta}}{ }^{\theta}
\end{aligned}
$$


parametrize superdiffeomorphisms generated by the vector field

$\Xi \cdot \partial \equiv \Xi^{z}(z, \bar{z}, \theta, \bar{\theta}) \partial_{z}+\Xi^{\bar{z}}(z, \bar{z}, \theta, \bar{\theta}) \partial_{\bar{z}}+\Xi^{\theta}(z, \bar{z}, \theta, \bar{\theta}) D_{\theta}+\Xi^{\bar{\theta}}(z, \bar{z}, \theta, \bar{\theta}) D_{\bar{\theta}}$

For convenience, we summarize the relations which hold for $H_{\theta}{ }^{z}=0$ :

$$
\begin{array}{rlrl}
\tau & =D \sqrt{\Lambda}, \quad H_{\theta}{ }^{\theta}=1, & H_{\bar{\theta}}{ }^{\theta}=-\frac{1}{2} D H_{\bar{\theta}}{ }^{2} & \text { and c.c. } \\
H_{\bar{z}}{ }^{z} & =\left(\bar{D}-H_{\bar{\theta}}{ }^{z} \partial\right) H_{\bar{\theta}}{ }^{z}+\left(H_{\bar{\theta}}{ }^{\theta}\right)^{2} \quad, \quad H_{\bar{z}}{ }^{\theta}=\frac{1}{2} D H_{\bar{z}}{ }^{z} & \text { and c.c. } .
\end{array}
$$

The integrating factor equations (IFEQ's) satisfied by the factor $\Lambda$ then reduce to

$$
\begin{aligned}
{\left[\bar{D}-H_{\bar{\theta}}{ }^{z} \partial-H_{\bar{\theta}}{ }^{\theta} D\right] \Lambda } & =\left(\partial H_{\bar{\theta}}{ }^{z}\right) \Lambda \\
{\left[\bar{\partial}-H_{\bar{z}}{ }^{z} \partial-H_{\bar{z}}{ }^{\theta} D\right] \Lambda } & =\left(\partial{H_{\bar{z}}}^{z}\right) \Lambda,
\end{aligned}
$$

the second equation being a consequence of the first one.

Under an infinitesimal superdiffeomorphism generated by the vector field $\Xi \cdot \partial$, the coordinates $(Z, \bar{Z}, \Theta, \bar{\Theta})$ change according to

$$
\begin{array}{lll}
s \Theta & =C^{z} \tau+C^{\theta} \sqrt{\Lambda} \equiv \mathcal{C}^{\Theta} & \text { and c.c. } \\
s Z & =C^{z} \Lambda-\Theta(s \Theta) \equiv \mathcal{C}^{Z}-\Theta s \Theta & \text { and c.c. } .
\end{array}
$$

Here and in the following, we assume that the ' $\Xi$ ' and ' $C$ ' are ghost fields and that ' $s$ ' is the BRS differential']. For $H_{\theta}{ }^{z}=0$, the ' $\Xi$ ' are restricted by the condition (9) and the induced variations of $H_{\bar{\theta}}{ }^{z}, \Lambda$ and $C^{z}$ read

$$
\begin{aligned}
s H_{\bar{\theta}}{ }^{z} & =\left[\bar{D}-H_{\bar{\theta}}^{z} \partial+\frac{1}{2}\left(D H_{\bar{\theta}}^{z}\right) D\right] C^{z}+\left(\partial H_{\bar{\theta}}{ }^{z}\right) C^{z} \\
s \Lambda & =\partial\left(C^{z} \Lambda\right)+\frac{1}{2}\left(D C^{z}\right) D \Lambda \\
s C^{z} & =-\left[C^{z} \partial C^{z}+\frac{1}{4}\left(D C^{z}\right)^{2}\right] .
\end{aligned}
$$

We note that the transformation law of $\Lambda$ can be rewritten by virtue of the IFEQ (12) as

$$
s \Lambda=\Xi \cdot \partial \Lambda+N \Lambda
$$

where

$$
N \equiv \partial \Xi^{z}+\left(\partial \Xi^{\bar{z}}\right) H_{\bar{z}}^{z}+\left(\partial \Xi^{\bar{\theta}}\right) H_{\bar{\theta}}^{z}
$$

\footnotetext{
${ }^{3}$ The $s$-operator is supposed to act from the right; the BRS-algebra is graded by the ghost number, the Grassmann parity being $s$-inert.
} 
On a SRS with $H_{\theta}{ }^{z}=0$, the chirally split form of the superdiffeomorphism anomaly is given by [3]

$\mathcal{A}_{D}\left[C^{z}, H_{\bar{\theta}}{ }^{z}\right]+$ c.c. $\equiv \frac{K}{2} \int_{\mathbf{S} \boldsymbol{\Sigma}} d^{4} z C^{z}\left[\partial^{2} D+3 \mathcal{R}_{z \theta} \partial+\left(D \mathcal{R}_{z \theta}\right) D+2\left(\partial \mathcal{R}_{z \theta}\right)\right] H_{\bar{\theta}}{ }^{z}+$ c.c.

where the superfield $\mathcal{R}_{z \theta}(z, \theta)$ is the component of a superprojective connection [7]. The WZ consistency conditions for the anomalies (7) and (16) read

$$
s \mathcal{A}_{W}\left[\Omega, \rho,{H_{\bar{\theta}}}^{z}\right]=0=s \mathcal{A}_{D}\left[C^{z}, H_{\bar{\theta}}{ }^{z}\right]
$$

Here, the $s$-operation is defined by eqs.(13)-(15) and by

$$
\begin{aligned}
s \Omega & =\Xi \cdot \partial \Omega \\
s \rho_{z} & =\Omega \rho_{z}+\Xi \cdot \partial \rho_{z}+N \rho_{z} \\
s \rho_{\bar{z}} & =\Omega \rho_{\bar{z}}+\Xi \cdot \partial \rho_{\bar{z}}+\bar{N} \rho_{\bar{z}} \\
s \mathcal{R}_{z \theta} & =0=s \overline{\mathcal{R}}_{\bar{z} \bar{\theta}}
\end{aligned}
$$

with $\Xi$ subject to eq.(9) and $\rho_{z}, \rho_{\bar{z}}$ related to $\rho_{Z}, \rho_{\bar{Z}}$ by

$$
\rho_{z}=\Lambda_{z}{ }^{Z} \rho_{Z} \quad, \quad \rho_{\bar{z}}=\bar{\Lambda}_{\bar{z}}^{\bar{Z}} \rho_{\bar{Z}} \text {. }
$$

Since we are dealing with generic SRS's (with $H_{\theta}{ }^{z}=0$ ), a comment should be made concerning the transformation laws of the basic variables under superconformal changes of coordinates $(z, \bar{z}, \theta, \bar{\theta}) \rightarrow\left(z^{\prime}, \bar{z}^{\prime}, \theta^{\prime}, \bar{\theta}^{\prime}\right)$ : these laws are given in terms of $\mathrm{e}^{-w} \equiv D \theta^{\prime}$ with $\bar{D} w=0$ and they read

$$
\begin{aligned}
\left(H_{\bar{\theta}}\right)^{\prime} & =\mathrm{e}^{\bar{w}} \mathrm{e}^{-2 w} H_{\bar{\theta}}{ }^{2} \quad, \quad\left(C^{z}\right)^{\prime}=\mathrm{e}^{-2 w} C^{z} \\
\left(\rho_{z}\right)^{\prime} & =\mathrm{e}^{2 w} \rho_{z} \quad, \quad\left(\rho_{\bar{z}}\right)^{\prime}=\mathrm{e}^{2 \bar{w}} \rho_{\bar{z}} \\
\left(\mathcal{R}_{z \theta}\right)^{\prime} & =\mathrm{e}^{3 w}\left[\mathcal{R}_{z \theta}-\mathcal{S}\left(z^{\prime}, \theta^{\prime} ; z, \theta\right)\right],
\end{aligned}
$$

where $\mathcal{S}$ denotes the super Schwarzian derivative.

\section{Liouville counterterm}

The WZ-action for the super Weyl anomaly is invariant under superdiffeomorphisms and therefore the last of eqs.([6) can be rewritten as

$$
s \Gamma_{W}[\phi, E]=-\mathcal{A}_{W}[\Omega, E],
$$

where $s$ is defined by the equations given above and by

$$
s \phi \equiv-\Omega+\Xi \cdot \partial \phi \text {. }
$$


The superfunction $\phi$ can be expressed in terms of the superconformal fields $\rho_{z}, \rho_{\bar{z}}$ introduced in eq. (18) and some background fields $\stackrel{\circ}{\rho}_{z}, \stackrel{\circ}{\rho}_{\bar{z}}$ which are $s$-inert $\left(s \stackrel{\circ}{\rho}_{z}=\right.$ $\left.0=s \stackrel{\circ}{\rho}_{\bar{z}}\right)$ :

$$
2 \phi \equiv \ln \stackrel{\circ}{\rho}_{z \bar{z}}-\ln \rho_{z \bar{z}}=\ln \stackrel{\circ}{\rho}_{Z \bar{Z}}-\ln \rho_{Z \bar{Z}}
$$

Here, $\stackrel{\circ}{\rho}_{z \bar{z}} \equiv \stackrel{\circ}{\rho}_{z} \stackrel{\circ}{\rho}_{\bar{z}}, \rho_{z \bar{z}} \equiv \rho_{z} \rho_{\bar{z}}$ and $\stackrel{\circ}{\rho}_{z}, \stackrel{\circ}{\rho}_{\bar{z}}$ are related to $\stackrel{\circ}{\rho}_{Z}, \stackrel{\circ}{\rho}_{\bar{Z}}$ as in eq.(18).

The Liouville counterterm is obtained by substituting eq.(22) into the expression (7) for the WZ-action :

$$
\begin{aligned}
\Gamma_{\text {Liouville }}[\rho, H ; \stackrel{\circ}{\rho}] & \equiv \Gamma_{W}[\phi, E] \\
= & \frac{K}{4} \int_{\mathbf{S} \boldsymbol{\Sigma}} d^{4} Z\left\{\left(\ln \rho_{Z \bar{Z}}-\ln \stackrel{\circ}{\rho}_{Z \bar{Z}}\right) D_{\Theta} D_{\bar{\Theta}}\left(\ln \rho_{Z \bar{Z}}-\ln \stackrel{\circ}{\rho}_{Z \bar{Z}}\right)\right. \\
& \left.+2\left(\ln \rho_{Z \bar{Z}}-\ln \stackrel{\circ}{\rho}_{Z \bar{Z}}\right) D_{\Theta} D_{\bar{\Theta}}\left(\ln \stackrel{\circ}{\rho}_{Z \bar{Z}}\right)\right\} .
\end{aligned}
$$

To evaluate the response of this functional to the $s$-variation induced by the transformations (13), we use the method outlined in appendix A of reference [3] from which it follows that

$$
\begin{array}{rlr}
s\left(d^{4} Z\right) & =d^{4} Z\left[\partial_{Z} \mathcal{C}^{Z}-D_{\Theta} \mathcal{C}^{\Theta}+\text { c.c. }\right] & \\
s \partial_{Z} & =-\left[\left(\partial_{Z} \mathcal{C}^{Z}\right) \partial_{Z}+\left(\partial_{Z} \mathcal{C}^{\bar{Z}}\right) \partial_{\bar{Z}}+\left(\partial_{Z} \mathcal{C}^{\Theta}\right) D_{\Theta}+\left(\partial_{Z} \mathcal{C}^{\bar{\Theta}}\right) D_{\bar{\Theta}}\right] \\
s D_{\Theta} & =-\left[\left(D_{\Theta} \mathcal{C}^{Z}\right) \partial_{Z}-2 \mathcal{C}^{\Theta} \partial_{Z}+\left(D_{\Theta} \mathcal{C}^{\bar{Z}}\right) \partial_{\bar{Z}}+\left(D_{\Theta} \mathcal{C}^{\Theta}\right) D_{\Theta}+\left(D_{\Theta} \mathcal{C}^{\bar{\Theta}}\right) D_{\bar{\Theta}}\right] & \text { and c.c. }
\end{array}
$$

Here, $\mathcal{C}^{Z}$ and $\mathcal{C}^{\Theta}$ are the parameters introduced in eqs.(13). By virtue of the previous relations and

$$
\mathcal{C}^{Z} \partial_{Z}+\mathcal{C}^{\Theta} D_{\Theta}+\text { c.c. }=\Xi \cdot \partial
$$

we find that

$s \Gamma_{\text {Liouville }}=-\mathcal{A}_{W}[\Omega, \rho, H]+\frac{K}{2} \int_{\mathbf{S} \mathbf{\Sigma}} d^{4} Z\left[\Xi \cdot \partial \ln \stackrel{\circ}{\rho}_{z \bar{z}}+N+\bar{N}\right] D_{\Theta} D_{\bar{\Theta}} \ln \stackrel{\circ}{\rho}_{Z \bar{Z}} \cdot$

The first term on the r.h.s. is the super Weyl anomaly and the second represents the non-chirally split form of the superdiffeomorphism anomaly: this term corresponds [17] [1] to a Weyl anomaly with $\rho$ replaced by $\stackrel{\circ}{\rho}$ and $\Omega$ replaced by the compensating value $\Omega_{\text {comp }}$ which is obtained by setting $\rho=\stackrel{\circ}{\rho}$ in the $s$-variation of $\ln \rho$ (see eqs.(17)):

$$
\Omega_{c o m p}=-\frac{1}{2}\left[\Xi \cdot \partial \ln \stackrel{\circ}{\rho}_{z \bar{z}}+N+\bar{N}\right] .
$$




\section{Passage to a chirally split expression}

The next (and most difficult) step consists of establishing a link between the integral on the r.h.s. of eq.(25) and a holomorphically factorized expression. This process involves the passage from the capital to the small coordinates and is technically very complicated. Therefore, we will only summarize the results and defer all details of the derivation to appendix C. The main ingredient of the calculation are the so-called 'intermediate' or 'tilde' coordinates $(\tilde{z}, \tilde{\bar{z}}, \tilde{\theta}, \tilde{\bar{\theta}}) \equiv$ $(Z, \bar{z}, \Theta, \bar{\theta})$ which mediate between the small and capital coordinates $[10$. The relevant equations are presented in appendix A and here we only note that these coordinates are not related to each other by complex conjugation.

By virtue of eqs.(18), (24), (12) and (10), the integral in eq.(25) can be rewritten as

$$
\begin{aligned}
\int_{\mathbf{S} \mathbf{\Sigma}} d^{4} Z\left[\Xi \cdot \partial \ln \stackrel{\circ}{\rho}_{z \bar{z}}+N+\bar{N}\right] D_{\Theta} D_{\bar{\Theta}} \ln \stackrel{\circ}{\rho}_{Z \bar{Z}}= & \int_{\mathbf{S} \boldsymbol{\Sigma}} d^{4} Z\left\{\left[\mathcal{C}^{Z} \partial_{Z}+\mathcal{C}^{\Theta} D_{\Theta}+\text { c.c. }\right] \ln \stackrel{\circ}{\rho}_{Z \bar{Z}}\right. \\
& \left.+\left[\frac{1}{\Lambda}\left(\partial \mathcal{C}^{Z}\right)+C^{\theta}(D \ln \Lambda)+\text { c.c. }\right]\right\} D_{\Theta} D_{\bar{\Theta}} \ln \stackrel{\circ}{\rho}_{Z \bar{Z}} .
\end{aligned}
$$

The variables $C^{\theta}$ and $\mathcal{C}^{\Theta}$ depend on the derivatives of $C^{z}$ according to eq.(9) from which it follows that

$$
\begin{aligned}
\mathcal{C}^{\Theta} & =\frac{1}{2 \sqrt{\Lambda}} D \mathcal{C}^{Z}=\frac{1}{2} \tilde{D} \mathcal{C}^{Z} \\
& =\frac{1}{2}\left[D_{\Theta}+\left(k_{\theta}{ }^{\bar{z}} \bar{L}\right) \partial_{\bar{Z}}+\left(k_{\theta} \overline{\bar{T}}+k_{\theta}{ }^{\bar{\theta}} \sqrt{\bar{L}}\right) D_{\bar{\Theta}}\right] \mathcal{C}^{Z}
\end{aligned}
$$

Here, the coefficients ' $k$ ' and the factors $\bar{L}, \bar{T}$ describe the passage from the tilde to the capital coordinates, see appendix A. As outlined in appendix C, the last equation and integration by parts allow us to recast the integral (26) in the form

$$
\begin{aligned}
\int_{\mathbf{S} \boldsymbol{\Sigma}} d^{4} Z \mathcal{C}^{Z} D_{\bar{\Theta}}\{[\tilde{\partial} & \left.+\frac{\tilde{\partial} k_{\bar{\theta}}^{\bar{z}}}{\left(k_{\bar{\theta}}^{\bar{\theta}}\right)^{2}}\left(\tilde{\bar{D}}-k_{\bar{\theta}} \bar{z} \tilde{\bar{\partial}}\right)\right] \stackrel{\circ}{\gamma}_{\Theta} \\
& -\frac{1}{2} \stackrel{\circ}{\Theta}_{\Theta}\left[\tilde{D}+\frac{\tilde{D} k_{\bar{\theta}}^{\bar{z}}}{\left(k_{\overline{\bar{\theta}}}^{\bar{\theta}}\right)^{2}}\left(\tilde{\bar{D}}-k_{\bar{\theta}} \overline{\bar{z}} \tilde{\bar{\partial}}\right)\right]{\stackrel{\circ}{\gamma_{\Theta}}}-\frac{1}{2}\left(k_{\theta} \overline{\bar{z}} \bar{L}\right)\left(D_{\bar{\Theta}} \stackrel{\circ}{\Theta}^{2}\right\}+\text { c.c., }
\end{aligned}
$$

where

$$
\stackrel{\circ}{\gamma}_{\Theta} \equiv D_{\Theta} \ln \stackrel{\circ}{\rho}_{Z \bar{Z}}
$$

From (18), (12) and the explicit form of the ' $k$ ', one concludes that the expression (29) is related to the background connection $\stackrel{\circ}{\gamma}_{\theta} \equiv D \ln \stackrel{\circ}{\rho}_{z \bar{z}}$ by

$$
\stackrel{\circ}{\gamma}_{\Theta}=\frac{1}{\sqrt{\Lambda}}(G-D \ln \Lambda)
$$


where $G$ equals $\stackrel{\circ}{\gamma}_{\theta}$ plus a contribution which does not depend on the integrating factors $\Lambda, \bar{\Lambda}$. Explicitly,

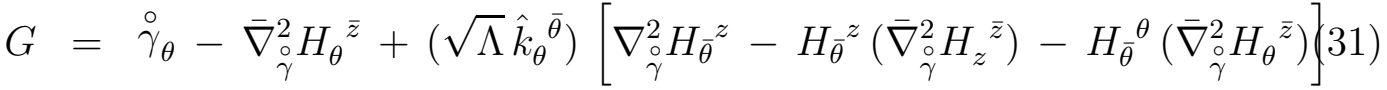

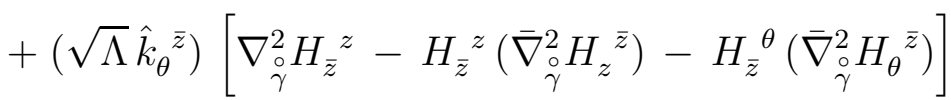

with $\hat{k}_{\theta}{ }^{\bar{\theta}}, \hat{k}_{\theta}{ }^{\bar{z}}$ given by eqs.(62) and $\nabla$ being the superconformally covariant derivative introduced in reference [7]:

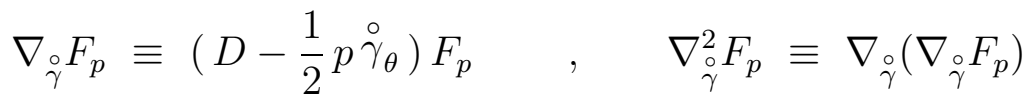

$\left(F_{p}=\right.$ conformal superfield of weight $p$ in the $(z, \theta)$-sector, e.g. $C^{z}$ has weight '-2'). In fact, eqs.(19) imply that $\stackrel{\circ}{\gamma}_{\theta}$ transforms like a (non-holomorphic) super affine connection under a superconformal change of coordinates:

$$
\left(\stackrel{\circ}{\gamma}_{\theta}\right)^{\prime}=\mathrm{e}^{w}\left[\stackrel{\circ}{\gamma}_{\theta}+2(D w)\right]
$$

Further use of the relations in appendix A yields the following expression for the integral (28):

$$
\int_{\mathbf{S} \mathbf{\Sigma}} d^{4} z \frac{C^{z}}{k_{\bar{\theta}}^{\bar{\theta}}}\left\{\nabla_{G}^{2} \psi-\left(\frac{\partial k_{\bar{\theta}}^{\bar{\theta}}}{k_{\bar{\theta}}^{\bar{\theta}}}-\frac{1}{2} G \frac{D k_{\bar{\theta}}^{\bar{\theta}}}{k_{\bar{\theta}}^{\bar{\theta}}}\right) \psi+\sqrt{\Lambda} k_{\theta}{ }^{\bar{z}} \psi\left[\bar{\partial} G-\nabla_{G}^{3} H_{\bar{z}}^{z}\right]+\sqrt{\Lambda} k_{\theta}{ }^{\bar{\theta}} \psi^{2}\right\}+\text { c.c.. }
$$

Here, $\nabla_{G}$ denotes the superconformally covariant derivative w.r.t. $G$ and the quantity

$$
\begin{aligned}
\psi & \equiv \frac{1}{k_{\bar{\theta}}^{\bar{\theta}}}\left\{\left[\bar{D} G+\nabla_{G}^{3} H_{\bar{\theta}}^{z}\right]-k_{\bar{\theta}}^{\bar{z}}\left[\bar{\partial} G-\nabla_{G}^{3} H_{\bar{z}}{ }^{z}\right]\right\} \\
& =\frac{1}{k_{\bar{\theta}} \bar{\theta}}\left\{\left[\partial D H_{\bar{\theta}}^{z}+k_{\bar{\theta}}^{\bar{z}} \partial D H_{\bar{z}}{ }^{z}\right]-\frac{1}{2}\left[\partial H_{\bar{\theta}}^{z}-k_{\bar{\theta}}^{\bar{z}} \partial H_{\bar{z}}^{z}\right] G+\left(\left(\overline{\bar{D}}-k_{\bar{\theta}} \bar{z} \overline{\bar{\partial}}\right) G\right\}\right.
\end{aligned}
$$

is independent of the integrating factors $\Lambda, \bar{\Lambda}$. Thereby, the integral (34) also has the property that it does not depend on $\Lambda$ and $\bar{\Lambda}$; this is a crucial point, since these factors are non-local functionals of ${H_{\bar{\theta}}}^{z}$ by virtue of the IFEQ's (12).

To summarize the derivation of this section, we have shown that the second term on the r.h.s. of eq.(25) has a chirally split form in the sense that it represents a linear functional of $C^{z}$ plus its complex conjugate. In the next section, this expression will be related to the chirally split superdiffeomorphism anomaly. 


\section{Passage to the chirally split superdiffeomor- phism anomaly: the second counterterm}

By substituting eqs.(31) and (35) into (34), we end up with the following expression for the integral on the r.h.s. of eq.(25):

$K \int_{\mathbf{S} \boldsymbol{\Sigma}} d^{4} z C^{z}\left\{\frac{1}{2} \partial^{2} D H_{\bar{\theta}}^{z}+\left[\bar{D}-H_{\bar{\theta}}^{z} \partial+\frac{1}{2}\left(D H_{\bar{\theta}}^{z}\right) D-\frac{3}{2}\left(\partial H_{\bar{\theta}}{ }^{z}\right)\right] \stackrel{\circ}{R}\right\}+$ c.c. $+M$

Here,

$$
\stackrel{\circ}{R} \equiv \stackrel{\circ}{R}_{z \theta} \equiv \frac{1}{2}\left[\partial \stackrel{\circ}{\gamma}_{\theta}-\frac{1}{2} \stackrel{\circ}{\gamma}_{\theta}\left(D \stackrel{\circ}{\gamma}_{\theta}\right)\right]
$$

represents a smooth superprojective connection [7] and we have only specified contributions coming from the leading term $\stackrel{\circ}{\gamma}_{\theta}$ of $G$; all other contributions are included in the quantity $M$, the latter being lengthy and not very illuminating, cf. appendix B.

Let us now consider the supersymmetric generalization of the second counterterm introduced in reference [1]:

$$
\Gamma_{I I} \equiv-K \int_{\mathbf{S} \Sigma} d^{4} z H_{\bar{\theta}}^{z}(\mathcal{R}-\stackrel{\circ}{R})+\text { c.c. }
$$

Here, $\mathcal{R} \equiv \mathcal{R}_{z \theta}$ and $\stackrel{\circ}{R} \equiv \stackrel{\circ}{R}_{z \theta}$ are holomorphic and smooth superprojective connections, respectively, as introduced in eqs.(16) and (37).

From eqs.(14) (16) and the $s$-invariance of $\mathcal{R}$ and $\stackrel{\circ}{\rho}_{z \bar{z}}$, it follows that

$s \Gamma_{I I}=\mathcal{A}_{D}-K \int_{\mathbf{S} \boldsymbol{\Sigma}} d^{4} z C^{z}\left\{\frac{1}{2} \partial^{2} D H_{\bar{\theta}}{ }^{2}+\left[\bar{D}-H_{\bar{\theta}}{ }^{z} \partial+\frac{1}{2}\left(D H_{\bar{\theta}}{ }^{z}\right) D-\frac{3}{2}\left(\partial H_{\bar{\theta}}{ }^{z}\right)\right] \stackrel{\circ}{R}\right\}+$ c.c. .

Obviously, the integrals in expressions (36) and (39) coincide with each other: combining eqs.(25) (36) and (39), we get

$$
\mathcal{A}_{W}[\Omega, E]+s\left(\Gamma_{\text {Liouville }}+\Gamma_{I I}\right)=\mathcal{A}_{D}\left[C^{z}, H_{\bar{\theta}}{ }^{z}\right]+\text { c.c. }+M \text {. }
$$

\section{Verlinde's functional and the third countert- erm}

The next step consists of determining the supersymmetric generalization of the Verlinde functional [8] on the superplane and then turning it into a globally welldefined action on a SRS by the inclusion of connection terms [1]. The final point (to be considered in the next section) is to check that the $s$-variation of this functional coincides with the quantity $M$ on the r.h.s. of eq.(40). 
To supersymmetrize the bosonic Verlinde action as defined on the complex plane $\mathbf{C}$, we express the latter in terms of the capital coordinates of the bosonic theoryf:

$$
\begin{aligned}
\Gamma_{\text {Verlinde }}[\mu, \bar{\mu}] & \equiv \int_{\mathbf{C}} d^{2} z \frac{1}{1-\mu \bar{\mu}}\left\{(\partial \mu)(\bar{\partial} \bar{\mu})-\frac{1}{2}\left[\bar{\mu}(\partial \mu)^{2}+\text { c.c. }\right]\right\} \\
& =\int_{\mathbf{C}} d^{2} Z\left\{\left(\partial_{\bar{Z}} \ln \lambda\right)\left(\partial_{Z} \ln \bar{\lambda}\right)-\frac{1}{2}\left[\bar{\mu} \frac{\bar{\lambda}}{\lambda}\left(\partial_{\bar{Z}} \ln \lambda\right)^{2}+\text { c.c. }\right]\right\} .
\end{aligned}
$$

Using this trick, we can immediately write down the graded generalization of the last expression on the superplane SC:

$$
\Gamma_{\text {Verlinde }}^{(\text {super }}\left[H_{\bar{\theta}}{ }^{z}, H_{\theta}{ }^{\bar{z}}\right]=\int_{\mathbf{S C}} d^{4} Z\left\{\left(D_{\bar{\Theta}} \ln \Lambda\right)\left(D_{\Theta} \ln \bar{\Lambda}\right)+\frac{1}{2}\left[H_{\theta}{ }^{\bar{z}} \frac{\bar{\Lambda}}{\sqrt{\Lambda}}\left(D_{\bar{\Theta}} \ln \Lambda\right)\left(\partial_{\bar{Z}} \ln \Lambda\right)+\text { c.c. }\right]\right\} \text {. }
$$

This functional can easily be expressed in terms of the small coordinates by virtue of eqs. (61) and the IFEQ's (59) (68):

$$
\begin{aligned}
& \Gamma_{\text {Verlinde }}^{(\text {super })}=\int_{\mathbf{S C}} d^{4} z \frac{1}{\left(k_{\bar{\theta}}\right)^{2}}\left\{[ \partial H _ { \overline { \theta } } ^ { z } - k _ { \overline { \theta } } ^ { \overline { z } } ( \partial H _ { \overline { z } } { } ^ { z } ) ] \left[\left[1+\left(\sqrt{\Lambda} \hat{k}_{\theta}^{\bar{\theta}}\right) H_{\bar{\theta}}{ }^{\theta}+\left(\sqrt{\Lambda} \hat{k}_{\theta}^{\bar{z}}\right) H_{\bar{z}}{ }^{\theta}\right] \bar{\partial} H_{\theta}^{\bar{z}}\right.\right. \\
& \left.+\left[\left(\sqrt{\Lambda} \hat{k}_{\theta}^{\bar{\theta}}\right) H_{\bar{\theta}}{ }^{z}+\left(\sqrt{\Lambda} \hat{k}_{\theta}^{\bar{z}}\right) H_{\bar{z}}{ }^{z}\right] \bar{\partial} H_{z}^{\bar{z}}\right] \\
& \left.\left.+\frac{1}{2}\left[\frac{1}{A} H_{\theta}^{\bar{z}}\left[\partial H_{\bar{\theta}}{ }^{z}-k_{\bar{\theta}} \bar{z}^{\left(\partial H_{\bar{z}}\right.}{ }^{z}\right)\right] \partial H_{\bar{z}}{ }^{z}+\text { c.c. }\right]\right\} \text {. }
\end{aligned}
$$

Here, the quantity $A$ is a function of the Beltrami coefficients (explicitly given by eq.(64) of appendix A). Although most of the ' $H$ ' and ' $k$ ' transform in a complicated way under superconformal transformations, it can be checked that the super Verlinde action (42) becomes globally well-defined on $\mathbf{S} \boldsymbol{\Sigma}$, if the derivatives appearing explicitly in this expression are replaced by supercovariant ones (see eqs.(70). This procedure provides the third counterterm $\Gamma_{I I I}\left[H_{\bar{\theta}}{ }^{z}, H_{\theta} \bar{z} ; \stackrel{\circ}{\rho}\right]$ :

$$
\begin{array}{r}
\Gamma_{I I I}=-K \int_{\mathbf{S} \Sigma} d^{4} z \frac{1}{\left(k_{\bar{\theta}}^{\bar{\theta}}\right)^{2}}\left\{[ \nabla _ { \gamma } ^ { 2 } H _ { \overline { \theta } } ^ { z } - k _ { \overline { \theta } } ^ { \overline { z } } ( \nabla _ { \gamma } ^ { 2 } H _ { \overline { z } } { } ^ { z } ) ] \left[\left[1+\left(\sqrt{\Lambda} \hat{k}_{\theta}^{\bar{\theta}}\right) H_{\bar{\theta}}{ }^{\theta}+\left(\sqrt{\Lambda} \hat{k}_{\theta}{ }^{\bar{z}}\right) H_{\bar{z}}{ }^{\theta}\right] \bar{\nabla}_{\gamma}^{2} H_{\theta}{ }^{\bar{z}}\right.\right. \\
\left.+\left[\left(\sqrt{\Lambda} \hat{k}_{\theta}{ }^{\bar{\theta}}\right) H_{\bar{\theta}}{ }^{z}+\left(\sqrt{\Lambda} \hat{k}_{\theta}{ }^{\bar{z}}\right) H_{\bar{z}}{ }^{z}\right] \bar{\nabla}_{\gamma}^{2} H_{z}{ }^{\bar{z}}\right] \\
\left.+\frac{1}{2}\left[\frac{1}{A} H_{\theta}^{\bar{z}}\left[\nabla_{\gamma}^{2} H_{\bar{\theta}}{ }^{z}-k_{\bar{\theta}}{ }^{\bar{z}}\left(\nabla_{\gamma}^{2} H_{\bar{z}}{ }^{z}\right)\right] \nabla_{\gamma}^{2} H_{\bar{z}}{ }^{z}+\text { c.c. }\right]\right\} .
\end{array}
$$

\section{Synthesis}

It remains to show that $s \Gamma_{I I I}$ coincides up to a sign with the quantity $M$ occurring on the r.h.s. of eq.(40). Since the $s$-variation of $H_{\bar{\theta}}{ }^{z}$ (and its c.c.) are known,

\footnotetext{
4 The latter are given in terms of reference coordinates $(z, \bar{z})$ by $d Z=$ $\lambda(z, \bar{z})[d z+\mu(z, \bar{z}) d \bar{z}]$.
} 
there is in principle no obstruction for determining the $s$-variations of the ' $k$ ' and of $\Gamma_{I I I}$. However, the latter expressions represent very complicated functions of $H_{\bar{\theta}}{ }^{z}$ and $H_{\theta}{ }^{\bar{z}}$ and therefore a general solution of this problem within a reasonable amount of time appears to be out of reach. For this reason, we rather solve this problem in the WZ-gauge. The corresponding equations are explicitly given below and our final result reads:

$$
\mathcal{A}_{W}[\Omega, E]+s\left(\Gamma_{\text {Liouville }}+\Gamma_{I I}+\Gamma_{I I I}\right)=\mathcal{A}_{D}\left[C^{z}, H_{\bar{\theta}}^{z}\right]+\text { c.c. } .
$$

It is unlikely that the validity of this result is destroyed by the inclusion of auxiliary fields which are absent in the WZ-gauge; in any case, the only possible modification consists of a term vanishing in the WZ-gauge (see also appendix B).

\section{Projection to component fields}

The super Weyl anomaly and the associated WZ action, as given by eqs.(6), are superspace integrals involving the supervielbein fields: the corresponding component field expressions immediately follow by application of the so-called density projection formula [1]].

The holomorphic superfield $\mathcal{R}$ admits a $\theta$-expansion of the form [7]

$$
\mathcal{R}_{z \theta}=\frac{i}{2} \chi_{z \theta}+\theta\left[\frac{1}{2} r_{z z}\right]
$$

where $\chi$ and $r$ only depend on $z$ and not on $\bar{z}$. All other component fields to appear in this section depend on both $z$ and $\bar{z}$.

In the WZ-supergauge, we have [10]

$$
H_{\bar{\theta}}^{z}=\bar{\theta} \mu_{\bar{z}}^{z}+\theta \bar{\theta}\left[-i \alpha_{\bar{z}}{ }^{\theta}\right] \quad, \quad C^{z}=c^{z}+\theta\left[i \epsilon^{\theta}\right] .
$$

Here, the space-time fields $\mu$ and $\alpha$ denote the Beltrami coefficient and its fermionic partner, respectively, while $c$ and $\epsilon$ parametrize ordinary diffeomorphisms and local supersymmetry transformations, respectively. In the following, we will simplify the notation by suppressing all indices on the component fields.

The $\theta$-expansions of the (real) supermetric $\stackrel{\circ}{\rho}_{z \bar{z}}$ and of the superaffine connection $\stackrel{\circ}{\gamma}_{\theta}$ read

$$
\begin{aligned}
& \stackrel{\circ}{\rho}_{z \bar{z}}=\stackrel{\circ}{\rho}_{0}+\theta\left[i \stackrel{\circ}{\rho}_{1}\right]+\bar{\theta}\left[-i \stackrel{\circ}{\rho}_{1}\right]+\theta \bar{\theta}\left[i \stackrel{\circ}{\rho}_{2}\right] \\
& \stackrel{\circ}{\gamma}_{\theta}=i \stackrel{\circ}{\eta}+\theta \stackrel{\circ}{\Gamma}+\bar{\theta} \stackrel{\circ}{\Upsilon}+\theta \bar{\theta}[-i \stackrel{\circ}{\tau}] .
\end{aligned}
$$

From these equations and the definition $\stackrel{\circ}{\gamma}_{\theta}=D \ln \stackrel{\circ}{\rho}_{z \bar{z}}$, we conclude that

$\stackrel{\circ}{\eta}=\stackrel{\circ}{\rho}_{1} / \stackrel{\circ}{\rho}_{0} \quad, \quad \stackrel{\circ}{\Gamma}=\partial \ln \stackrel{\circ}{\rho}_{0} \quad, \quad \stackrel{\circ}{\Upsilon}=i\left(\stackrel{\circ}{\rho}_{0}\right)^{-1}\left[\stackrel{\circ}{\rho}_{2}-\frac{i}{2} \stackrel{\circ}{\rho}_{1} \stackrel{\circ}{\rho}_{1} / \stackrel{\circ}{\rho}_{0}\right] \quad, \quad \stackrel{\circ}{\tau}=\partial \stackrel{\circ}{\eta}$. 
By virtue of eq.(33), the space-time field $\stackrel{\circ}{\Upsilon}$ transforms homogeneously under superconformal changes of coordinates and thereby we can choose it to vanish in the WZ-gauge: according to the last set of equations, this simply amounts to a redefinition of $\stackrel{\circ}{\rho}_{2}$ in terms of the other components of the supermetric $\stackrel{\circ}{\rho}_{z \bar{z}}$. Thus, we are left with $\stackrel{\circ}{\Gamma}_{\equiv} \stackrel{\circ}{\Gamma}_{z}$ (which represents a non-holomorphic affine connection) and its fermionic partner $\stackrel{\circ}{\eta} \equiv \stackrel{\circ}{\eta}_{\theta}$ as well as the complex conjugate variables.

From the defining relation $\stackrel{\circ}{R}_{z \theta}=\frac{1}{2}\left[\partial \stackrel{\circ}{\gamma}_{\theta}-\frac{1}{2} \stackrel{\circ}{\gamma}_{\theta}\left(D \stackrel{\circ}{\gamma}_{\theta}\right)\right]$ and the expansion

$$
\stackrel{\circ}{R}_{z \theta}=\frac{i}{2} \stackrel{\circ}{\chi}+\theta\left[\frac{1}{2} \stackrel{\circ}{r}\right]+\bar{\theta}\left[\frac{1}{2} \stackrel{\circ}{u}\right]+\theta \bar{\theta}\left[-\frac{i}{2} \stackrel{\circ}{\kappa}\right]
$$

it follows that

$$
\begin{aligned}
& \stackrel{\circ}{\chi}=\left(\partial-\frac{1}{2} \stackrel{\circ}{\Gamma} \stackrel{\circ}{\eta} \equiv \chi(\stackrel{\circ}{\Gamma})\right. \\
& \stackrel{\circ}{r}=\left(\partial \stackrel{\circ}{\Gamma}-\frac{1}{2} \stackrel{\circ}{\Gamma}^{2}\right)-\frac{1}{2} \stackrel{\circ}{\eta} \chi(\stackrel{\circ}{\Gamma}) \equiv r(\stackrel{\circ}{\Gamma})-\frac{1}{2} \stackrel{\circ}{\eta} \chi(\stackrel{\circ}{\Gamma}) \equiv t(\stackrel{\circ}{\Gamma}) .
\end{aligned}
$$

Here, we introduced the functions $\chi(\cdot), r(\cdot)$ and $t(\cdot)$ which may be viewed as 'field strengths' and which render the formulae below compact and transparent.

The supercovariant derivatives in superspace project down to similar derivatives in space-time:

$$
\begin{aligned}
\left(\nabla_{\gamma}^{2} H_{\bar{z}}{ }^{z}\right) \mid & =(\partial+\stackrel{\circ}{\Gamma}) \mu+\frac{1}{2} \stackrel{\circ}{\eta} \alpha \equiv \mathcal{D} \mu \\
\left(\nabla_{\gamma}^{3} H_{\bar{z}}{ }^{z}\right) \mid & =i\left[\left(\partial+\frac{1}{2} \stackrel{\circ}{\Gamma}\right) \alpha+\left(\frac{1}{2} \stackrel{\circ}{\eta} \partial+(\partial \stackrel{\circ}{\eta})\right) \mu\right] \equiv i \mathcal{D} \alpha .
\end{aligned}
$$

These expressions represent the 'field strengths' of $\mu$ and $\alpha$ and they appear for instance in the $\theta$-component of $G$ as defined by eq.(31):

$$
(D G) \mid=\stackrel{\circ}{\Gamma}-\frac{1}{1-\mu \bar{\mu}}[\overline{\mathcal{D}} \bar{\mu}-\bar{\mu} \mathcal{D} \mu] \equiv g .
$$

The symmetry transformations of the basic space-time fields follow from the superspace variations (14),

$$
\begin{aligned}
s \mu & =[\bar{\partial}-\mu \partial+(\partial \mu)] c+\frac{1}{2} \alpha \epsilon \\
s \alpha & =\left[\bar{\partial}-\mu \partial+\frac{1}{2}(\partial \mu)\right] \epsilon+c \partial \alpha-\frac{1}{2} \alpha \partial c \\
s c & =-c \partial c+\frac{1}{4} \epsilon \epsilon \\
s \epsilon & =-c \partial \epsilon-\frac{1}{2} \epsilon \partial c,
\end{aligned}
$$

where we have the following assignments: 


\begin{tabular}{||l||c|c|c|c||}
\hline \hline Field & $\mu$ & $\alpha$ & $c$ & $\epsilon$ \\
\hline Grassmann parity & 0 & 1 & 0 & 1 \\
\hline Ghost number & 0 & 0 & 1 & 1 \\
\hline \hline
\end{tabular}

As to the action of the $s$-operator, we adhere to the conventions specified in the footnote in section 3. The $s$-operator defined by eqs.(53) is then nilpotent by construction.

Substitution of the $\theta$-expansions into our previous superspace results yields

$$
\begin{aligned}
\mathcal{A}_{D}= & -\frac{K}{2} \int_{\Sigma} d^{2} z\left\{c\left[\left[\partial^{3}+2 r \partial+(\partial r)\right] \mu+\left[\frac{3}{2} \chi \partial+\frac{1}{2}(\partial \chi)\right] \alpha\right]\right. \\
\left.-\epsilon\left[\left[\partial^{2}+\frac{1}{2} r\right] \alpha+\left[\frac{3}{2} \chi \partial+(\partial \chi)\right] \mu\right]\right\} & -\alpha . c . \\
\Gamma_{I I}= & -\frac{K}{2} \int_{\Sigma} d^{2} z\{\mu(r-\stackrel{\circ}{r})-\alpha(\chi-\stackrel{\circ}{\chi})\}+\text { c.c. } \\
\Gamma_{I I I}= & \frac{K}{2} \int_{\Sigma} d^{2} z \frac{1}{1-\mu \bar{\mu}}\left\{(\mathcal{D} \mu)(\overline{\mathcal{D}} \bar{\mu})-\frac{1}{2} \bar{\mu}(\mathcal{D} \mu)^{2}-\frac{1}{2} \mu(\overline{\mathcal{D}} \bar{\mu})^{2}\right\} .
\end{aligned}
$$

Using the $s$-transformations of $\mu$ and $\alpha$, the $s$-invariance of $\stackrel{\circ}{\Gamma}$ and $\stackrel{\circ}{\eta}$ as well as some integration by parts, we find that

$$
\begin{aligned}
s \Gamma_{I I I}= & \frac{K}{2} \int_{\Sigma} d^{2} z\{(s \mu)[r(g)-r(\stackrel{\circ}{\Gamma})]-(s \alpha)[\chi(g)-\chi(\stackrel{\circ}{\Gamma})]\}+\text { c.c. } \\
=\frac{K}{2} \int_{\Sigma} d^{2} z & \left\{c\left[-[\bar{\partial}-\mu \partial-2(\partial \mu)][r(g)-r(\stackrel{\circ}{\Gamma})]-\left[\frac{1}{2} \alpha \partial+\frac{3}{2}(\partial \alpha)\right][\chi(g)-\chi(\stackrel{\circ}{\Gamma})]\right]\right. \\
& \left.\quad-\epsilon\left[\frac{1}{2}[r(g)-r(\stackrel{\circ}{\Gamma})] \alpha-\left[\bar{\partial}-\mu \partial-\frac{3}{2}(\partial \mu)\right][\chi(g)-\chi(\stackrel{\circ}{\Gamma})]\right]\right\}+ \text { c.c. , }
\end{aligned}
$$

where $g$ was defined in eq.(52) and where $\chi(\cdot)$ and $r(\cdot)$ refer to the notation introduced in eqs.(50).

In the WZ-gauge, the expression (34) takes the form

$$
\begin{array}{r}
\operatorname{expression}(34)=-\int_{\Sigma} d^{2} z\left\{c\left[\partial^{3} \mu-[\bar{\partial}-\mu \partial-2(\partial \mu)] t(g)-\left[\frac{1}{2} \alpha \partial+\frac{3}{2}(\partial \alpha)\right] \chi(g)\right]\right. \\
\left.-\epsilon\left[\left[\partial^{2}+\frac{1}{2} t(g)\right] \alpha-\left[\bar{\partial}-\mu \partial-\frac{3}{2}(\partial \mu)\right] \chi(g)\right]\right\}+ \text { c.c. } \quad,(56)
\end{array}
$$

where $t(\cdot)$ was defined in eq.(50). Furthermore, equation (39) becomes

$$
\begin{array}{r}
s \Gamma_{I I}=\mathcal{A}_{D}+\frac{K}{2} \int_{\Sigma} d^{2} z\left\{c\left[\partial^{3} \mu-[\bar{\partial}-\mu \partial-2(\partial \mu)] \stackrel{\circ}{r}-\left[\frac{1}{2} \alpha \partial+\frac{3}{2}(\partial \alpha)\right] \stackrel{\circ}{\chi}\right]\right. \\
\left.-\epsilon\left[\left[\partial^{2}+\frac{1}{2} \stackrel{\circ}{r}\right] \alpha-\left[\bar{\partial}-\mu \partial-\frac{3}{2}(\partial \mu)\right] \stackrel{\circ}{\chi}\right]\right\}+ \text { c.c. . }
\end{array}
$$

Since the expression (56) represents the WZ-gauge version of the integral on the r.h.s. of eq.(25), it is readily seen from the latter formula and eqs.(55) (57) that the result (44) holds in the WZ-gauge. In particular, it encompasses the results of the bosonic theory [1]. 


\section{Conclusion}

By virtue of a technical tour de force, we have constructed the local counterterm relating the super Weyl anomaly with the chirally split superdiffeomorphism anomaly (as defined on a compact SRS). This result explicitly proves the equivalence of both anomalies which expressions have been known for some time and discussed in the literature. As by-products of our construction, we obtained the non-chirally split superdiffeomorphism anomaly and the super Verlinde action which are of independent interest. The combination of the second and third counterterm can be used to derive a holomorphic factorization theorem for arbitrary central charge along the lines of reference [2]. This derivation involves a discussion of renormalized determinants and the index theorem for families and is to be discussed separately 18 .

\section{Acknowledgements}

J.-P.A. is grateful to the Theory Division of CERN for their hospitality and financial support during a stay in autumn 1991 where part of this work has been done. F.G. wishes to thank the Theory Group of Bordeaux for their hospitality at an early stage of this work. 


\section{A Intermediate coordinates}

In the following, we recall the basic relations for the so-called intermediate or tilde coordinates [10] and we derive some useful equations which find a direct application in the main text.

The tilde coordinates are introduced in analogy to the capital coordinates by considering smooth transformations,

$$
(z, \bar{z}, \theta, \bar{\theta}) \longrightarrow(\tilde{z}, \tilde{\bar{z}}, \tilde{\theta}, \tilde{\bar{\theta}}) \equiv(Z, \bar{z}, \Theta, \bar{\theta}) \longrightarrow(Z, \bar{Z}, \Theta, \bar{\Theta})
$$

They are not related to each other by complex conjugation. For $H_{\theta}{ }^{z}=0$ (and c.c.), we have the relations

$$
\begin{aligned}
\tilde{\partial} & =\frac{1}{\Lambda}[\partial-(D \ln \sqrt{\Lambda}) D] \\
\tilde{\bar{\partial}} & =\bar{\partial}-H_{\bar{z}}{ }^{z} \partial-H_{\bar{z}}{ }^{\theta} D \\
\tilde{D} & =\frac{1}{\sqrt{\Lambda}} D \\
\tilde{\bar{D}} & =\bar{D}-H_{\bar{\theta}}{ }^{z} \partial-H_{\bar{\theta}}{ }^{\theta} D,
\end{aligned}
$$

by virtue of which the IFEQ's (12) take the compact form

$$
\tilde{\bar{D}} \ln \Lambda=\partial H_{\bar{\theta}}{ }^{z} \quad, \quad \tilde{\bar{\partial}} \ln \Lambda=\partial H_{\bar{z}}{ }^{z} .
$$

Quite generally, the tilde and capital coordinates are related by 10

$$
\begin{aligned}
\tilde{\partial} & =\partial_{Z}+\left(k_{z}^{\bar{z}} \bar{L}\right) \partial_{\bar{Z}}+\left(k_{z}^{\bar{z}} \bar{T}+k_{z}^{\bar{\theta}} \sqrt{\bar{L}}\right) D_{\bar{\Theta}} \\
\tilde{\bar{\partial}} & =\bar{L} \partial_{\bar{Z}}+\bar{T} D_{\bar{\Theta}} \\
\tilde{D} & =D_{\Theta}+\left(k_{\theta}{ }^{\bar{z}} \bar{L}\right) \partial_{\bar{Z}}+\left(k_{\theta} \overline{\bar{T}}+k_{\theta} \bar{\theta} \sqrt{\bar{L}}\right) D_{\bar{\Theta}} \\
\tilde{\bar{D}} & =\left(k_{\bar{\theta}} \overline{\bar{T}}+k_{\bar{\theta}} \overline{\bar{\theta}} \sqrt{\bar{L}}\right) D_{\bar{\Theta}}+\left(k_{\bar{\theta}} \overline{\bar{z}} \bar{L}\right) \partial_{\bar{Z}},
\end{aligned}
$$

or

$$
\begin{aligned}
\partial_{Z} & =\tilde{\partial}-\hat{k}_{z}{ }^{\bar{z}} \tilde{\bar{\partial}}-\hat{k}_{z}^{\bar{\theta}} \tilde{\bar{D}} \\
\partial_{\bar{Z}} & =\frac{1}{\bar{L}}\left[\tilde{\bar{\partial}}-\frac{\bar{T}}{\sqrt{\bar{L}} k_{\bar{\theta}}^{\bar{\theta}}}\left(\tilde{\bar{D}}-k_{\bar{\theta}}{ }^{\bar{z}} \tilde{\bar{\partial}}\right)\right] \\
D_{\Theta} & =\tilde{D}-\hat{k}_{\theta}^{\bar{z}} \tilde{\bar{\partial}}-\hat{k}_{\theta}^{\bar{\theta}} \tilde{\bar{D}} \\
D_{\bar{\Theta}} & =\frac{1}{\sqrt{\bar{L}} k_{\bar{\theta}}^{\bar{\theta}}}\left[\tilde{\bar{D}}-k_{\bar{\theta}}{ }^{\bar{z}} \tilde{\bar{\partial}}\right]
\end{aligned}
$$

with

$$
\begin{array}{ll}
\hat{k}_{z}^{\bar{\theta}} \equiv\left(k_{\bar{\theta}}^{\bar{\theta}}\right)^{-1} k_{z}^{\bar{\theta}} & , \quad \hat{k}_{z}^{\bar{z}} \equiv k_{z}^{\bar{z}}-\hat{k}_{z}^{\bar{\theta}} k_{\bar{\theta}}^{\bar{z}} \\
\hat{k}_{\theta}^{\bar{\theta}} \equiv\left(k_{\bar{\theta}}^{\bar{\theta}}\right)^{-1} k_{\theta} \bar{\theta} & , \quad \hat{k}_{\theta}^{\bar{z}} \equiv k_{\theta}{ }^{\bar{z}}-\hat{k}_{\theta}^{\bar{\theta}} k_{\bar{\theta}}^{\bar{z}} .
\end{array}
$$


For $H_{\theta}{ }^{z}=0$ (and c.c.), we have the explicit expressions

$$
\begin{aligned}
& \bar{L}=\bar{\Lambda} A \quad, \quad k_{\bar{\theta}}^{\bar{z}}=-A^{-1}\left(H_{\bar{\theta}}{ }^{z} H_{z}{ }^{\bar{z}}+H_{\bar{\theta}}{ }^{\theta} H_{\theta}{ }^{\bar{z}}\right) \\
& k_{\theta}{ }^{\bar{z}}=\Lambda^{-1 / 2} A^{-1} H_{\theta}{ }^{\bar{z}} \quad, \quad k_{z}{ }^{\bar{z}}=\Lambda^{-3 / 2} A^{-1}\left(H_{z}{ }^{\bar{z}} \sqrt{\Lambda}+H_{\theta}{ }^{\bar{z}} \tau\right) \\
& \bar{T}=A \bar{\tau}-\left(H_{\bar{z}}{ }^{z} H_{z}{ }^{\bar{\theta}}+H_{\bar{z}}{ }^{\theta} H_{\theta}{ }^{\bar{\theta}}\right) \sqrt{\bar{\Lambda}} \\
& k_{z}^{\bar{\theta}}=\Lambda^{-3 / 2} A^{-1 / 2}\left[\left(H_{z}^{\bar{\theta}} \sqrt{\Lambda}-H_{\theta}{ }^{\bar{\theta}} \tau\right)+A^{-1}\left(H_{z}{ }^{\bar{z}} \sqrt{\Lambda}+H_{\theta}{ }^{\bar{z}} \tau\right)\left(H_{\bar{z}}{ }^{z} H_{z} \bar{\theta}+H_{\bar{z}}{ }^{\theta} H_{\theta}{ }^{\bar{\theta}}\right)\right] \\
& k_{\theta}^{\bar{\theta}}=\Lambda^{-1 / 2} A^{-1 / 2}\left[H_{\theta}^{\bar{\theta}}+A^{-1} H_{\theta}^{\bar{z}}\left(H_{\bar{z}}{ }^{z} H_{z}{ }^{\bar{\theta}}+H_{\bar{z}}{ }^{\theta} H_{\theta}{ }^{\bar{\theta}}\right)\right] \\
& k_{\bar{\theta}}^{\bar{\theta}}=A^{-1 / 2}\left[1-\left(H_{\bar{\theta}}{ }^{\theta} H_{\theta}{ }^{\bar{\theta}}+H_{\bar{\theta}}{ }^{z} H_{z}^{\bar{\theta}}\right)-A^{-1}\left(H_{\bar{\theta}}{ }^{z} H_{z}^{\bar{z}}+H_{\bar{\theta}}{ }^{\theta} H_{\theta}{ }^{\bar{z}}\right)\left(H_{\bar{z}}^{z} H_{z}^{\bar{\theta}}+H_{\bar{z}}{ }^{\theta} H_{\theta}{ }^{\bar{\theta}}\right)\right],
\end{aligned}
$$

where

$$
A \equiv 1-\left(H_{\bar{z}}{ }^{z} H_{z}{ }^{\bar{z}}+H_{\bar{z}}{ }^{\theta} H_{\theta}{ }^{\bar{z}}\right)
$$

The Jacobians for the transformations (58) and (61) are given by $\sqrt{\Lambda}$ and $\sqrt{\bar{L}} / k_{\bar{\theta}} \bar{\theta}$, respectively.

From the structure relations $\left\{D_{\Theta}, D_{\Theta}\right\}=2 \partial_{Z},\left\{D_{\bar{\Theta}}, D_{\bar{\Theta}}\right\}=2 \partial_{\bar{Z}}$, all other graded commutators $=0$, one can derive useful relations between the ' $k$ ' and $\bar{L}, \bar{T}$, e.g.

$$
\begin{aligned}
& D_{\bar{\Theta}} k_{\bar{\theta}}^{\overline{\bar{\theta}}}=\frac{1}{\sqrt{\bar{L}} k_{\bar{\theta}}^{\bar{\theta}}}\left[1-\left(k_{\bar{\theta}}^{\bar{\theta}}\right)^{2}\right] \quad, \quad D_{\bar{\Theta}} k_{\bar{\theta}}^{\bar{\theta}}=-\frac{1}{2 \sqrt{\bar{L}}} \tilde{\bar{\partial}} k_{\bar{\theta}}^{\bar{z}} \\
& D_{\bar{\Theta}}\left(\frac{1}{\sqrt{\bar{L}}}\right)=-\frac{1}{\bar{L}}\left[\frac{\bar{T}}{\sqrt{\bar{L}}}+\frac{\tilde{\bar{\partial}} k_{\bar{\theta}}^{\bar{z}}}{2 k_{\bar{\theta}} \bar{\theta}}\right] \quad, \quad \tilde{\bar{D}}\left(\frac{k_{\bar{\theta}}^{\bar{z}}}{\left(k_{\bar{\theta}} \overline{\bar{\theta}}\right)^{2}}\right)=\frac{1}{\left(k_{\bar{\theta}}\right)^{2}}\left[1-\left(k_{\bar{\theta}}^{\bar{\theta}}\right)^{2}\right] \\
& \left(k_{\bar{\theta}}{ }^{\bar{\theta}}\right)^{2}=1-\tilde{\bar{D}} k_{\bar{\theta}}^{\bar{z}}+k_{\bar{\theta}} \overline{\bar{z}} \tilde{\bar{\partial}} k_{\bar{\theta}}^{\bar{z}} \quad, \quad\left(\tilde{\bar{D}}-k_{\bar{\theta}} \bar{z} \tilde{\bar{\partial}}\right)^{2}=\left(k_{\bar{\theta}}^{\bar{\theta}}\right)^{2} \tilde{\bar{\partial}} \\
& D_{\Theta} \hat{k}_{\theta}^{\bar{z}}=\hat{k}_{z}^{\bar{z}}+\left(\hat{k}_{\theta}^{\bar{\theta}}\right)^{2} \quad, \quad D_{\Theta} \hat{k}_{\theta}{ }^{\bar{\theta}}=\hat{k}_{z}{ }^{\bar{\theta}}
\end{aligned}
$$

and

$$
\begin{aligned}
D_{\bar{\Theta}}\left(k_{z}^{\bar{z}} \bar{L}\right)-2\left(k_{z}^{\bar{z}} \bar{T}+k_{z} \bar{\theta} \sqrt{\bar{L}}\right) & =\sqrt{\bar{L}} \frac{\tilde{\partial} k_{\bar{\theta}}^{\bar{z}}}{k_{\bar{\theta}}^{\bar{\theta}}} \\
D_{\bar{\Theta}}\left(k_{\theta} \overline{\bar{z}} \bar{L}\right)+2\left(k_{\theta} \overline{\bar{z}} \bar{T}+k_{\theta} \bar{\theta} \sqrt{\bar{L}}\right) & =-\sqrt{\bar{L}} \frac{\tilde{D} k_{\bar{\theta}}^{\bar{z}}}{k_{\bar{\theta}}^{\bar{\theta}}} .
\end{aligned}
$$

The IFEQ's (59) allow us to evaluate $D_{\Theta} \ln \bar{\Lambda}$. The resulting expression involves some lengthy terms proportional to $\bar{D} \ln \bar{\Lambda}$ and $\bar{\partial} \ln \bar{\Lambda}$. However, these terms vanish by virtue of the relations between the ' $k$ ' and ' $H$ ' and one is left with a differential polynomial in the ' $H$ ' (up to a factor $1 / \sqrt{\Lambda}$ ):

$D_{\Theta} \ln \bar{\Lambda}=\frac{1}{\sqrt{\Lambda}}\left\{\left[1+\left(\sqrt{\Lambda} \hat{k}_{\theta}^{\bar{\theta}}\right) H_{\bar{\theta}}^{\theta}+\left(\sqrt{\Lambda} \hat{k}_{\theta}^{\bar{z}}\right) H_{\bar{z}}^{\theta}\right] \bar{\partial} H_{\theta}^{\bar{z}}+\left[\left(\sqrt{\Lambda} \hat{k}_{\theta}^{\bar{\theta}}\right) H_{\bar{\theta}}^{z}+\left(\sqrt{\Lambda} \hat{k}_{\theta}^{\bar{z}}\right) H_{\bar{z}}{ }^{z}\right] \bar{\partial} H_{z}^{\bar{z}}\right\}$. 
The complex conjugate expression immediately follows from eqs.(61)(59) and (63):

$$
D_{\bar{\Theta}} \ln \Lambda=\frac{1}{\sqrt{\bar{\Lambda}}}\left\{\frac{1}{\sqrt{A} k_{\bar{\theta}}^{\bar{\theta}}}\left[\partial H_{\bar{\theta}}^{z}-k_{\bar{\theta}}^{\bar{z}} \partial H_{\bar{z}}^{z}\right]\right\} .
$$

Since the ' $k$ ' and the dependent ' $H$ ' involve derivatives of the basic variable $H_{\bar{\theta}}{ }^{z}$, they transform in a complicated, non-homogenous way under a superconformal change of coordinates, eqs.(19). Fortunately, the functionals of interest to us (like the super Verlinde action (42)) involve combinations of these variables which transform in a simple way:

$$
\begin{aligned}
A^{\prime} & =A\left[1+(\bar{D} \bar{w}) k_{\bar{\theta}}^{\bar{z}}\right], \quad\left(k_{\bar{\theta}}^{\bar{\theta}}\right)^{\prime}=k_{\bar{\theta}}^{\bar{\theta}}+\frac{1}{2}(\bar{D} \bar{w}) A^{-1 / 2} k_{\bar{\theta}}^{\bar{z}}\left(1-H_{\bar{\theta}}{ }^{\theta} H_{\theta}{ }^{\bar{\theta}}-H_{\bar{\theta}}{ }^{z} H_{z}^{\bar{\theta}}\right) \\
\left(\sqrt{\Lambda} \hat{k}_{\theta}^{\bar{z}}\right)^{\prime} & =\mathrm{e}^{w} \mathrm{e}^{-2 \bar{w}}\left(\sqrt{\Lambda} \hat{k}_{\theta}^{\bar{z}}\right) \quad, \quad\left(k_{\bar{\theta}}^{\bar{z}}\right)^{\prime}=\mathrm{e}^{-\bar{w}} k_{\bar{\theta}}^{\bar{z}} \\
\left(\sqrt{\Lambda} \hat{k}_{\theta}^{\bar{\theta}}\right)^{\prime} & =\mathrm{e}^{w} \mathrm{e}^{-\bar{w}}\left[\left(\sqrt{\Lambda} \hat{k}_{\theta}^{\bar{\theta}}\right)+(\bar{D} \bar{w})\left(\sqrt{\Lambda} \hat{k}_{\theta}^{\bar{z}}\right)\right] .
\end{aligned}
$$

\section{B Relating small and capital coordinates}

As in the main text, we consider the case $H_{\theta}{ }^{z}=0$ (and c.c.). The particular relation between the small and capital coordinates as expressed by eqs.(61) implies that a functional written in terms of the capital coordinates takes a particular form when expressed in terms of the small coordinates: generically, one obtains an integral of the form

$$
\int d^{4} z \frac{1}{\left(k_{\bar{\theta}}^{\bar{\theta}}\right)^{2}}\left[\mathcal{P}\left(H_{\bar{\theta}}^{z}\right)+k_{\bar{\theta}}^{\bar{z}} \mathcal{P}\left(H_{\bar{z}}^{z}\right)\right],
$$

where $\mathcal{P}\left(H_{\bar{\theta}}{ }^{z}\right)$ denotes the action of a differential operator $\mathcal{P}$ on $H_{\bar{\theta}}{ }^{z}$. For instance, for the super $b c$-system [4], the authors of reference [10] found that

$$
\int d^{4} Z \mathcal{B}_{\Theta Z} D_{\bar{\Theta}} \mathcal{C}^{Z}=-\int d^{4} z \frac{1}{\left(k_{\bar{\theta}}\right)^{2}} B_{\theta z}\left[s H_{\bar{\theta}}^{z}-k_{\bar{\theta}}^{\bar{z}} s H_{\bar{z}}^{z}\right],
$$

where $s$ denotes the BRS operator and

$$
\mathcal{B}_{\Theta Z}=\Lambda^{-3 / 2} B_{\theta z} \quad, \quad \mathcal{C}^{Z}=\Lambda C^{z} .
$$

Further examples are provided by the derivations in the main body of the text and will explicitly be given below.

By virtue of eq.(11), we can express $H_{\bar{z}}{ }^{z}$ in terms of the independent variable $H_{\bar{\theta}}{ }^{z}$ as $H_{\bar{z}}{ }^{z}=\tilde{\bar{D}} H_{\bar{\theta}}^{z}-1 / 4\left(D H_{\bar{\theta}}^{z}\right)^{2}$ and use integration by parts to transform $\mathcal{P}\left(H_{\bar{z}}{ }^{z}\right)$ into an expression involving $\mathcal{P}\left(H_{\bar{\theta}}^{z}\right)$. Application of the relation between $k_{\bar{\theta}} \bar{\theta}$ and $k_{\bar{\theta}} \bar{z}$ given by eqs. (66) then yields

$$
\int d^{4} z \frac{1}{\left(k_{\bar{\theta}}^{\bar{\theta}}\right)^{2}}\left[\mathcal{P}\left(H_{\bar{\theta}}{ }^{z}\right)+k_{\bar{\theta}}^{\bar{z}} \mathcal{P}\left(H_{\bar{z}}{ }^{z}\right)\right]=\int d^{4} z \mathcal{P}\left(H_{\bar{\theta}}{ }^{z}\right)+\int d^{4} z \frac{k_{\bar{\theta}}^{\bar{z}}}{\left(k_{\bar{\theta}}^{\bar{\theta}}\right)^{2}}[\ldots],
$$


where the second term vanishes in the WZ-gauge. E.g. the expression (72) can be cast into the form

$$
-\int d^{4} z B_{\theta z} s H_{\bar{\theta}}^{z}-\int d^{4} z \frac{k_{\bar{\theta}}^{\bar{z}}}{\left(k_{\bar{\theta}}^{\bar{\theta}}\right)^{2}}\left[\tilde{\bar{D}} B_{\theta z}-\frac{3}{2}\left(\partial H_{\bar{\theta}}^{z}\right) B_{\theta z}\right]\left(s H_{\bar{\theta}}{ }^{z}\right)
$$

and, in the WZ-gauge, this quantity equals $\int d^{2} z\left\{b_{z z} s \mu+\beta_{\theta z} s \alpha\right\}$ where we used the $\theta$-expansion $B_{\theta z}=i \beta_{\theta z}+\theta b_{z z}+\ldots$

Let us now come to the examples encountered in the present paper. By substituting eqs.(31) and (35) into (34), we end up with the following expression for the integral on the r.h.s. of eq. (25):

$$
\begin{aligned}
K \int_{\mathbf{S} \boldsymbol{\Sigma}} d^{4} z & \frac{1}{\left(k_{\bar{\theta}}^{\bar{\theta}}\right)^{2}}\left(C^{z}\left\{\frac{1}{2} \partial^{2} D H_{\bar{\theta}}{ }^{z}+\left[\bar{D}-H_{\bar{\theta}}{ }^{z} \partial+\frac{1}{2}\left(D H_{\bar{\theta}}^{z}\right) D-\frac{3}{2}\left(\partial H_{\bar{\theta}}{ }^{z}\right)\right] \stackrel{\circ}{R}\right\}\right. \\
& \left.+k_{\bar{\theta}}{ }^{\bar{z}} C^{z}\left\{\frac{1}{2} \partial^{2} D H_{\bar{z}}{ }^{z}-\left[\bar{\partial}-H_{\bar{z}}{ }^{z} \partial-\frac{1}{2}\left(D H_{\bar{z}}{ }^{z}\right) D-\frac{3}{2}\left(\partial H_{\bar{z}}{ }^{z}\right)\right] \stackrel{\circ}{R}\right\}\right)+ \text { c.c. }+\ldots \\
= & s \Gamma_{X}+\left[\mathcal{A}_{X}+\text { c.c. }\right]+\ldots .
\end{aligned}
$$

Here,

$$
\begin{array}{r}
\mathcal{A}_{X}\left[C^{z}, H_{\bar{\theta}}{ }^{z}\right] \equiv \frac{K}{2} \int_{\mathbf{S} \boldsymbol{\Sigma}} d^{4} z \frac{1}{\left(k_{\bar{\theta}}^{\bar{\theta}}\right)^{2}} C^{z}\left\{\left[\partial^{2} D+3 \mathcal{R} \partial+(D \mathcal{R}) D+2(\partial \mathcal{R})\right] H_{\Phi^{2}}{ }^{2} 6\right) \\
\left.+k_{\bar{\theta}}{ }^{\bar{z}}\left[\partial^{2} D+3 \mathcal{R} \partial+(D \mathcal{R}) D+2(\partial \mathcal{R})\right] H_{\bar{z}}{ }^{z}\right\}
\end{array}
$$

and

$$
\Gamma_{X} \equiv K \int_{\mathbf{S} \mathbf{\Sigma}} d^{4} z \frac{1}{\left(k_{\bar{\theta}}^{\bar{\theta}}\right)^{2}}\left[H_{\bar{\theta}}^{z}-k_{\bar{\theta}}^{\bar{z}} H_{\bar{z}}^{z}\right](\mathcal{R}-\stackrel{\circ}{R})+\text { c.c. } .
$$

Clearly, the expressions (75)-(77) are all of the form (71). By the procedure outlined above, we find

$$
\begin{aligned}
& \mathcal{A}_{X}=\mathcal{A}_{D}+\mathcal{A}_{Q} \\
& \mathcal{A}_{Q} \equiv \frac{K}{2} \int_{\mathbf{S} \Sigma} d^{4} z \frac{k_{\bar{\theta}}^{\bar{z}}}{\left(k_{\bar{\theta}}^{\bar{\theta}}\right)^{2}}\left\{\left(s H_{\bar{\theta}}^{z}\right)\left[\partial^{2} D+3 \mathcal{R} \partial+(D \mathcal{R}) D+2(\partial \mathcal{R})\right] H_{\bar{\theta}}{ }^{z}\right. \\
& \left.-2 C^{z} H_{\bar{\theta}}{ }^{z}\left[\left(\partial H_{\bar{\theta}}{ }^{z}\right)(\partial \mathcal{R})-\left(D H_{\bar{\theta}}{ }^{z}\right)(\partial D \mathcal{R})\right]\right\} \quad,
\end{aligned}
$$

(where $\mathcal{A}_{D}$ is the chirally split superdiffeomorphism anomaly, eq.(16)) and

$$
\begin{aligned}
& \Gamma_{X}=-\Gamma_{I I}-\Gamma_{P} \\
& \Gamma_{P} \equiv K \int_{\mathbf{S} \Sigma} d^{4} z \frac{k_{\bar{\theta}}^{\bar{z}}}{\left(k_{\bar{\theta}}^{\bar{\theta}}\right)^{2}}\left\{\tilde{\bar{D}}(\mathcal{R}-\stackrel{\circ}{R}) H_{\bar{\theta}}^{z}-\frac{1}{2}(\mathcal{R}-\stackrel{\circ}{R})\left[H_{\bar{\theta}}^{z}\left(\partial H_{\bar{\theta}}^{z}\right)+\frac{1}{2}\left(D H_{\bar{\theta}}^{z}\right)^{2}\right]\right\}+\text { c.c. , }
\end{aligned}
$$

where $\Gamma_{I I}$ represents the counterterm introduced in eq. 38 ). Furthermore, the integral in eq. (75) can be rewritten as

$$
\begin{gathered}
K \int_{\mathbf{S} \boldsymbol{\Sigma}} d^{4} z C^{z}\left\{\frac{1}{2} \partial^{2} D H_{\bar{\theta}}^{z}+\left[\bar{D}-H_{\bar{\theta}}^{z} \partial+\frac{1}{2}\left(D H_{\bar{\theta}}{ }^{z}\right) D-\frac{3}{2}\left(\partial H_{\bar{\theta}}{ }^{z}\right)\right] \stackrel{\circ}{R}\right\} \\
+K \int_{\mathbf{S} \boldsymbol{\Sigma}} d^{4} z \frac{k_{\bar{\theta}}^{\bar{z}}}{\left(k_{\bar{\theta}}^{\bar{\theta}}\right)^{2}}\left(s H_{\bar{\theta}}^{z}\right)\left\{\frac{1}{2} \partial^{2} D H_{\bar{\theta}}^{z}+\left[\bar{D}-H_{\bar{\theta}}^{z} \partial+\frac{1}{2}\left(D H_{\bar{\theta}}^{z}\right) D-\frac{3}{2}\left(\partial H_{\bar{\theta}}{ }^{z}\right)\right] \stackrel{\circ}{R}\right\},
\end{gathered}
$$


where the first contribution is the one specified in eq.(36).

\section{Technical details}

In this appendix, we provide some computational details for the derivations of section 5 .

From eqs.(29), (65), (27), (60), (13), (58) and integration by parts, it follows that the expression (26) can be rewritten (up to the 'c.c.' contribution) as

$$
\begin{aligned}
\int_{\mathbf{S} \Sigma} d^{4} Z \mathcal{C}^{Z} D_{\bar{\Theta}}\left\{\left[\tilde{\partial}+\left[D_{\bar{\Theta}}\left(k_{z}^{\bar{z}} \bar{L}\right)-2\left(k_{z}^{\bar{z}} \bar{T}+k_{z}^{\bar{\theta}} \sqrt{\bar{L}}\right)\right] D_{\bar{\Theta}}\right]{\stackrel{\circ}{\gamma_{\Theta}}}^{(81)}\right. \\
\left.-\frac{1}{2} \stackrel{\circ}{\Theta}_{\Theta}\left[\tilde{D}-\left[D_{\bar{\Theta}}\left(k_{\theta} \bar{L} \bar{L}\right)+2\left(k_{\theta}^{\bar{z}} \bar{T}+k_{\theta}^{\bar{\theta}} \sqrt{\bar{L}}\right)\right] D_{\bar{\Theta}}\right] \stackrel{\circ}{\Theta}_{\Theta}-\frac{1}{2}\left(k_{\theta}{ }^{\bar{z}} \bar{L}\right)\left(D_{\bar{\Theta}} \stackrel{\circ}{\Theta}_{\Theta}\right)^{2}\right\} .
\end{aligned}
$$

Next, substitution of (67) and (60) into the previous integral yields the expression (28) (up to the c.c.).

The passage between eqs.(29) and (30) gives rise to complicated expressions which are proportional to $\stackrel{\circ}{\bar{\theta}}_{\bar{\theta}}, \bar{D} \gamma_{\bar{\theta}}^{\circ}, \bar{D} \ln \bar{\Lambda}$ and $\bar{\partial} \ln \bar{\Lambda}$, respectively. Each of these expressions can be shown to vanish by virtue of the relations between $\bar{L}, \bar{T}$ and the ' $k$ ' which follow from the structure equations.

The derivation of eq.(34) from (28) relies on the formulae (66) and on the commutation relations between $D_{\bar{\Theta}}$ and the tilde derivatives. 


\section{References}

[1] M.Knecht, S.Lazzarini and F.Thuillier, Phys.Lett. B251 (1990) 279.

[2] M.Knecht, S.Lazzarini and R.Stora, Phys.Lett. B262 (1991) 25.

[3] F.Delduc and F.Gieres, Int.J.Mod.Phys. A7 (1992) 1685.

[4] D.Friedan, in "Unified String Theories", Santa Barbara Workshop, M.B.Green and D.Gross, eds. (World Scientific, 1986) ;

D.Friedan, E.Martinec and S.Shenker, Nucl.Phys. B271 (1986) 93 ;

L.Crane and J.M.Rabin, Commun.Math.Phys. 113 (1988) 601 ;

M.Batchelor and P.Bryant, Commun.Math.Phys. 114 (1988) 243 ;

A.M.Baranov, Yu.I.Manin, I.V.Frolov and A.S.Schwarz, Commun.Math.Phys. 111 (1987) 373 .

[5] A.M.Polyakov, Phys.Lett. 103B (1981) 211;

E.Martinec, Phys.Rev. D28 (1983) 2604;

M.Lauer, Class. Quantum Grav. 4 (1987) 781.

[6] F.Gieres and J.McCabe, Phys.Lett. B202 (1988) 339 .

[7] F.Gieres, "Conformally covariant operators on Riemann surfaces (with applications to conformal and integrable models)", preprint CERNTH.5985/91 .

[8] H.Verlinde, Nucl.Phys. B337 (1990) 652 .

H.Verlinde And E.VerLinde, "Conformal field theory and geometric quantization", in "Superstrings", Proceedings Trieste 1989.

[9] J. De Boer and J.Goeree, Phys.Lett. B274 (1992) 289.

[10] F.Delduc And F.Gieres, Class. Quantum Grav. 7 (1990) 1907.

[11] S.J.Gates,JR. And H.Nishino, Class. Quantum Grav. 3 (1986) 391 ;

R.Brooks and S.J.Gates,JR., Nucl.Phys. B287 (1987) 669 ;

S.J.Gates,Jr. And F.Gieres, Nucl.Phys. B320 (1989) 310 .

[12] M.Roček, P.van Nieuwenhuizen and S.C.Zhang, Ann.Phys. 172 (1986) 348 ;

M.Evans, J.Louis And B.A.Ovrut, Phys.Rev. D35 (1987) 3045 . 
[13] R.Grimm, Nucl.Phys.(Proc.Suppl.) B5 (1988) 137 ;

L.Baulieu, M.Bellon and R.Grimm, Nucl.Phys. B321 (1989) 697 ;

R.Grimm, Ann.Phys., NY 200 (1990) 49.

[14] J.Wess And J.BAGger, "Supersymmetry and Supergravity" (Princeton University Press, 1983) ;

S.J.Gates, Jr., M.T.Grisaru, M.RoČEK And W.Siegel, "Superspace" (Benjamin/Cummings, 1983) .

[15] P.Howe, J.Phys. A12 (1979) 443.

[16] F.Gieres, Gen.Rel.Grav. 22 (1990) 889 ;

F.GIERES, "Geometry of supersymmetric gauge theories", Lecture Notes in Physics 302 (Springer Verlag, 1988) .

[17] L.Baulieu, C.Becchi and R.Stora, Phys.Lett. B180 (1986) 55 .

[18] F.Gieres, "On the holomorphic factorization in superconformal field theory", in preparation. 